\title{
New Insight on the Influence of Chemistry on Nucleation and Growth in High Temperature Superconducting Wires Through Spectrum Imaging
}

\author{
D.J. Miller,* N.J. Zaluzec,* X. Li,** S. Sathyamurthy,** M. Rupich** \\ * Materials Science Division, Argonne National Laboratory, Argonne, IL 60439 \\ ** American Superconductor Corporation, Devens, MA 01434
}

One successful approach for the manufacture of rare-earth cuprate superconductor (REBCO) wires is based on a post-deposition ex situ conversion of metal-organic deposited (MOD) precursors on textured substrate templates.[1,2] In the conversion step, the precursor crystallizes to the superconducting phase with initial nucleation at the template followed by homoepitaxial growth through the thickness of the coating. The deposition of multiple coatings is often used to achieve thicker conductor layers that can carry more current. However, a chemically segregated layer referred to as the "interlayer" - invariably forms at the interface between coatings in these multi-coat films. This chemically-segregated interlayer disrupts the propagation of the growth front and causes a loss of texture quality in the succeeding layer.[3] In this work using electron beam imaging and elemental mapping, we identified a mechanism by which the REBCO grows past the interlayer in two-layer films that also provides an explanation for degraded texture above the interlayer.

In order to study the conversion and growth process, double-layer precursor films were quenched at various points in the process, and the partially converted films were examined in cross-section by SEM and TEM/STEM. Cross-sections for both TEM and SEM were produced by focused-ion-beam techniques. Fig. 1 shows an SEM cross-section of a film in which the conversion to REBCO has completed through the bottom layer but for the most part has not yet proceeded into the top layer. At the interface between the layers an interlayer region consisting of various phases is observed, as marked with arrows in the region marked "A." In this region, the light phase below that layer is fully converted REBCO. Above that layer, the film remains unconverted. The spectral images in Fig. 2 show the chemical segregation at the interlayer and reveal that the interlayer consists of a mixture of $\mathrm{Ba}-(\mathrm{O}, \mathrm{F})$ and $\mathrm{Cu}-\mathrm{O}$ (light elements not shown) capped by a thin layer of RE-oxide. The specific identify of the phases comprising the interlayer have been identified through a variety of techniques including spectroscopy and electron diffraction. Fig. 3 shows spectral images of three films quenched at slightly different times in the conversion process. These images that combine Ba and $\mathrm{Cu}$ signals show that as the REBCO growth front moves past the interlayer, REBCO growth takes place by dissolution of the $\mathrm{Cu}-\mathrm{O}$ phase while the $\mathrm{Ba}-(\mathrm{O}, \mathrm{F})$ phase remains relatively intact. Thus, a mechanism by which the REBCO surpasses the interlayer involves growth through a "channel" in the interlayer, followed by the resumption of lateral growth on top of the interlayer. This mechanism can be observed in the region labeled "B" in Fig. 1. The arrow indicates a region in which the REBCO has surpassed the interlayer and it is evident that the REBCO visible above the interlayer grew through a channel in the interlayer and then spread laterally atop the interlayer.

This growth mechanism, revealed by spectrum imaging, provides an explanation for the degradation of current-carrying capacity for the REBCO above the interlayer. The lateral growth of the REBCO above the interlayer does not take place by homoepitaxial growth on pre-existing REBCO. Thus, small deviations in orientation can develop leading to poorer texture and a decrease in performance. 
References

[1] M.W. Rupich, et al. Physica C 412 (2004) 887-884

[2] T. Izumi, et al. IEEE Trans. Appl. Superconductivity 19 (2009) 3119-3122

[3] D.J. Miller, et al. US DOE High Temperature Superconductivity Program 2009 Peer Review. Alexandria, VA (2009), available online at: www.htspeerreview.com/agenda.html

[4] Research supported by the U.S. DOE, Office of Electricity Delivery and Energy Reliability, and Office of Science - Basic Energy Science under contract DE-AC02-06CH11357.

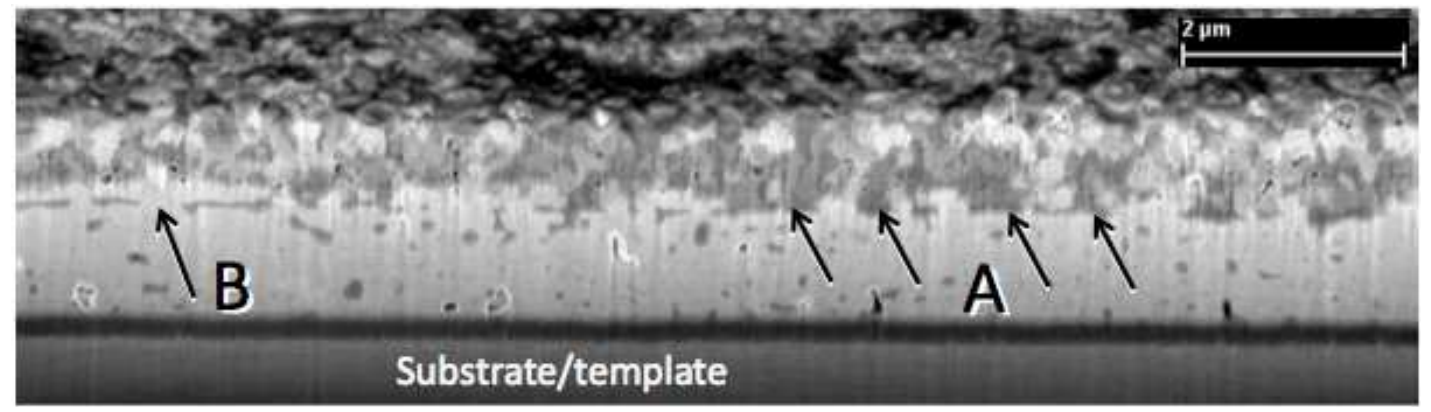

FIG. 1. Cross-sectional scanning electron micrograph of a partially converted REBCO film. In region $\mathrm{A}, \mathrm{REBCO}$ extends from the template to the interlayer (marked by arrows). Above the interlayer the film remains unconverted. In region B, REBCO has grown past the interlayer.
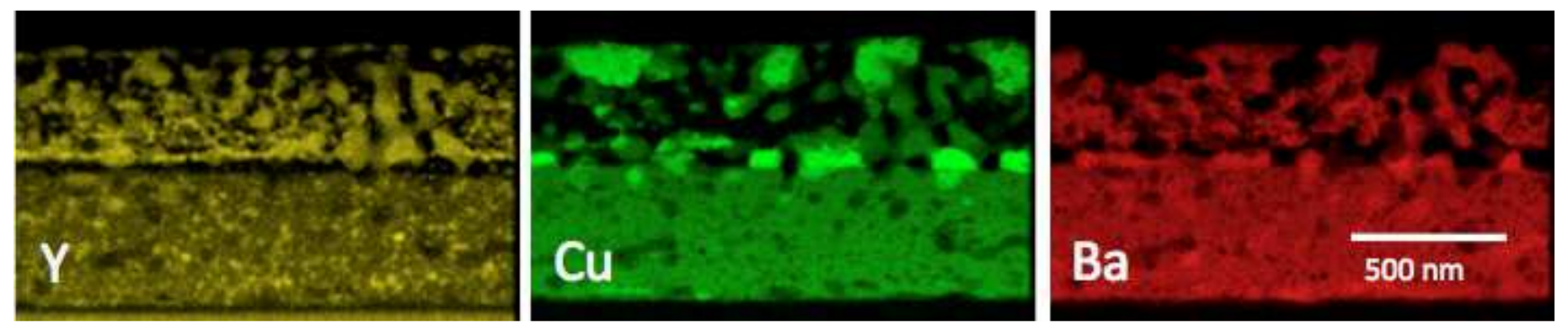

FIG. 2. Spectral images revealing the chemical segregation at the interlayer.
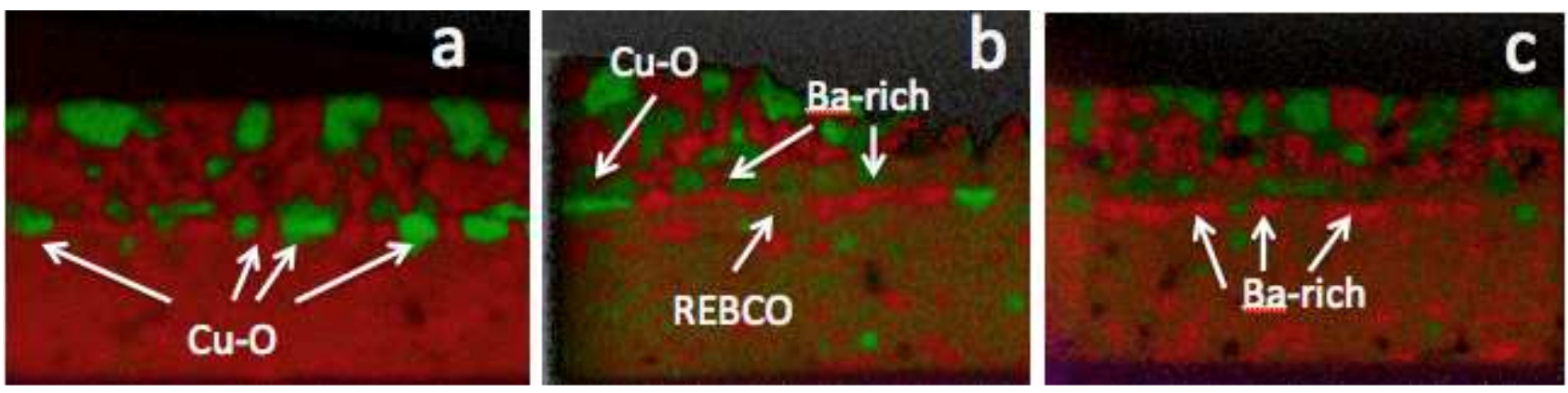

FIG. 3. Correlation maps of $\mathrm{Ba}$ and $\mathrm{Cu}$ of films quenched at various points in the conversion process. In (a) no conversion has taken place. The interlayer consists primarily of $\mathrm{Cu}-\mathrm{O}$ and $\mathrm{Ba}$-rich phases. In (b), the REBCO (mottled contrast) has reached and just begun to penetrate the interlayer. Note the absence of $\mathrm{Cu}-\mathrm{O}$ at the point of penetration (marked by arrow) while the Ba-rich phases remain distinct. In (c) REBCO has completely surpassed the interlayer. Again, note the absence of $\mathrm{Cu}-\mathrm{O}$ due to dissolution by the growing REBCO. 